\title{
Hyperpolarized Singlet NMR on a Small Animal Imaging System
}

\author{
Christoffer Laustsen, ${ }^{1,2,3}$ Giuseppe Pileio, ${ }^{4}$ Michael C. D. Tayler, ${ }^{4}$ Lynda J. Brown, ${ }^{4}$ \\ Richard C. D. Brown, ${ }^{4}$ Malcolm H. Levitt, ${ }^{4}$ and Jan H. Ardenkjaer-Larsen ${ }^{1,3,5 *}$
}

\begin{abstract}
Nuclear spin hyperpolarization makes a significant advance toward overcoming the sensitivity limitations of in vivo magnetic resonance imaging, particularly in the case of low-gamma nuclei. The sensitivity may be improved further by storing the hyperpolarization in slowly relaxing singlet populations of spin$1 / 2$ pairs. Here, we report hyperpolarized ${ }^{13} \mathrm{C}$ spin order transferred into and retrieved from singlet spin order using a small animal magnetic resonance imaging scanner. For spins in sites with very similar chemical shifts, singlet spin order is sustained in high magnetic field without requiring strong radiofrequency irradiation. The demonstration of robust singlet-to-magnetization conversion, and vice versa, on a small animal scanner, is promising for future in vivo and clinical deployments. Magn Reson Med 000:000-000, 2012. ( 2012 Wiley Periodicals, Inc.

Key words: DNP; hyperpolarization; singlet state
\end{abstract}

\section{INTRODUCTION}

Dynamic nuclear hyperpolarization (DNP) (1) is a major advance in nuclear magnetic resonance (NMR) and magnetic resonance imaging (MRI), overcoming one of the main weaknesses, which is the low signal strength. Applications of hyperpolarized spin order to the in vivo imaging of metabolism are already widespread (2). However, it remains that hyperpolarized magnetization is limited to the timescale of the spin-lattice relaxation $\left(T_{1}\right)$, which is usually less than $1 \mathrm{~min}$. In this article, we explore the use of pairs of magnetic nuclei to extend the lifetime of hyperpolarization by exploiting the properties of nuclear singlet states $(3,4)$. Molecules containing two spin-1/2 nuclei may exist as singlet and triplet nuclear spin isomers; namely molecules that have identical molecular structure but differ in their nuclear magnetism. The singlet spin isomer results from the subtraction of the angular momenta and is nonmagnetic (total spin $I=0$ ); that is, it denotes the quantum state

${ }^{1}$ Danish Research Centre for Magnetic Resonance, Copenhagen University Hospital Hvidovre, Hvidovre, Denmark.

${ }^{2}$ The MR Research Centre, Department of Clinical Medicine, Aarhus University Hospital, Aarhus, Denmark.

${ }^{3}$ Department of Electrical Engineering, Technical University of Denmark, Lyngby, Denmark.

${ }^{4}$ School of Chemistry, University of Southampton, Southampton, United Kingdom.

${ }^{5}$ GE Healthcare, Broendby, Denmark.

${ }^{*}$ Correspondence to: Jan H. Ardenkjaer-Larsen, Danish Research Centre for Magnetic Resonance, Hvidovre Hospital, Hvidovre, Denmark. E-mail: Jan. Henrik.Ardenkjaer-Larsen@ge.com

Received 2 May 2012; revised 25 June 2012; accepted 29 June 2012.

DOI 10.1002/mrm.24430

Published online in Wiley Online Library (wileyonlinelibrary.com).

(c) 2012 Wiley Periodicals, Inc.
$\left|S_{0}\right\rangle=(|\alpha \beta\rangle-|\beta \alpha\rangle) / \sqrt{2}$, where the polarizations of the nuclei are opposite with respect to an external quantization axis. By adding the angular momenta, the triplet spin isomer is formed (total spin $I=1$ ). The triplet exhibits nuclear paramagnetism with the component spins parallel, encompassing the three quantum states $(|\alpha \beta\rangle-|\beta \alpha\rangle) / \sqrt{2},|\alpha \alpha\rangle$, and $|\beta \beta\rangle$.

Nuclear magnetization is identified with the triplet, the only spin isomer with overall magnetic moment, and only triplet-triplet transitions are capable of generating observable NMR signal. Although it cannot be directly observed by NMR, the singlet isomer plays an active and useful role within the magnetic resonance experiment. The singlet is capable of maintaining spin order over timescales much longer $(3,5)$ than the "conventional" spin-lattice relaxation time, $T_{1}$, the upper time limit to the decay of the triplet manifold $(6,7)$. This occurs because singlet-triplet transitions are usually slower compared to triplet-triplet transitions. If coherent singlet-triplet transitions can be suppressed, the possibility arises of storing magnetization within the slowly decaying singlet, and retrieving it at a later time $t>>T_{1}$ for any conventional purpose, for example, acquiring an image. Singlet and triplet states interconvert when the two nuclei experience different magnetic environments.

If the nuclei have identical chemical shifts, that is, are in equivalent molecular sites, the singlet and triplet are disconnected. In this limit, singlet order is difficult to access. To access the singlet order, one must have nuclei in magnetically distinct sites. This may occur, when the nuclei have different chemical shifts. Magnetic inequivalence, however, may require that additional steps are taken to suppress the singlet-triplet interconversion during storage periods. Current methods include applying radiofrequency spin locking fields $(5,8,9)$, performing a chemical reaction (10), or rapidly shuttling the sample into a region of low magnetic field $(3,4)$.

These methods are usually impractical in a clinical context. In this article, we demonstrate singlet order storage and retrieval on a sample containing molecules in which the two ${ }^{13} \mathrm{C}$ spins are strongly coupled, that is, have a spin-spin scalar coupling constant whose magnitude exceeds the size of the chemical shielding frequency difference between the two nuclear sites. Nearly equivalent spin pairs confer several benefits in vivo, which we have reported in previous work (11):

1. The spin eigenstates of a strongly coupled pair are close to the singlet and triplet spin isomers. In this 
regime, the coherent singlet-triplet transitions are very weak, and do not require artificial suppression.

2. The pulse sequence for triplet to singlet conversion can operate efficiently in spite of the nonideal $B_{0}$ and $B_{1}$ magnetic fields produced by imaging hardware. The singlet-triplet interconversion involves applying trains of spin echoes that are insensitive to magnetic field inhomogeneity across the sample volume. Chemical site selective pulses are not required. Simple yet effective phase-cycling schemes are put in place to protect against radiofrequency field offsets, and poorly calibrated flip angles. These features ensure robust and efficient operation on an in vivo detection setup.

We demonstrate singlet order storage and retrieval on a hardware setup typical of an in vivo dissolution-DNP laboratory with no further hardware than the polarizer and MR scanner required. Hyperpolarized magnetization (nuclear triplet order) is produced in a sample containing coupled ${ }^{13} \mathrm{C}$ spin pairs. This sample is subsequently transferred to a small animal MR scanner, whereupon the hyperpolarization is transferred into singlet spin order solely using a radiofrequency pulse sequence. The singlet order is reconverted after some waiting period, again by a pulse sequence, for observation. We observe the resulting NMR signals persisting over a timescale longer than $T_{1}$.

\section{METHODS}

\section{Sample Preparation}

The experiments were carried out on a sample of cyclohexyl-isopropyl-1,2- ${ }^{13} \mathrm{C}_{2}$-oxalate- $\mathrm{d}_{18}$ synthesized in-house (Fig. 1). A detailed procedure will be reported elsewhere.

\section{Equipment}

The MRI experiments were carried out on a 4.7T preclinical horizontal bore magnet (Oxford Instruments, Oxford, UK) equipped with a direct drive console (Varian, Palo Alto, CA). The acquisition software was VNMRJ 2.3A (Agilent, Palo Alto, CA). A dual tuned ${ }^{1} \mathrm{H} /{ }^{13} \mathrm{C}$ volume transmit coil and an actively decoupled ${ }^{13} \mathrm{C}$ surface receive coil (RAPID Biomedical GmbH, Würzburg, Germany) were used. The hyperpolarization was carried out with a HyperSense polarizer (Oxford Instruments Molecular Biotools, Oxford, UK).

\section{Hyperpolarization}

A 30.6-mM stock solution of radical was prepared by combining $24.4 \mathrm{mg}(15.3 \mu \mathrm{mol})$ of the proprietary trityl radical AH111501 (GE Healthcare, Little Chalfont, UK) with 500 $\mu \mathrm{L}$ (562 mg) of dimethyl sulfoxide (DMSO). This stock solution (100 $\mu \mathrm{L}, 112 \mathrm{mg}$ ) was added to $20 \mathrm{mg}$ of cyclohexylisopropyl-1,2- ${ }^{13} \mathrm{C}_{2}$-oxalate- $\mathrm{d}_{18}$ dissolved in $50 \mu \mathrm{L}$ of methanol. This corresponds to approximately a $15 \mathrm{mM}$ radical concentration and a $10 \% \mathrm{w} / \mathrm{w}$ concentration of oxalate.

The hyperpolarization (dissolution-DNP) was performed as described in Ref. 1. A typical hyperpolarization experiment involved placing $100 \mu \mathrm{L}$ of the oxalate mixture into the polarizer sample holder and cooling to $1.4 \mathrm{~K}$. The sample was then irradiated with $100-\mathrm{mW}$ microwave
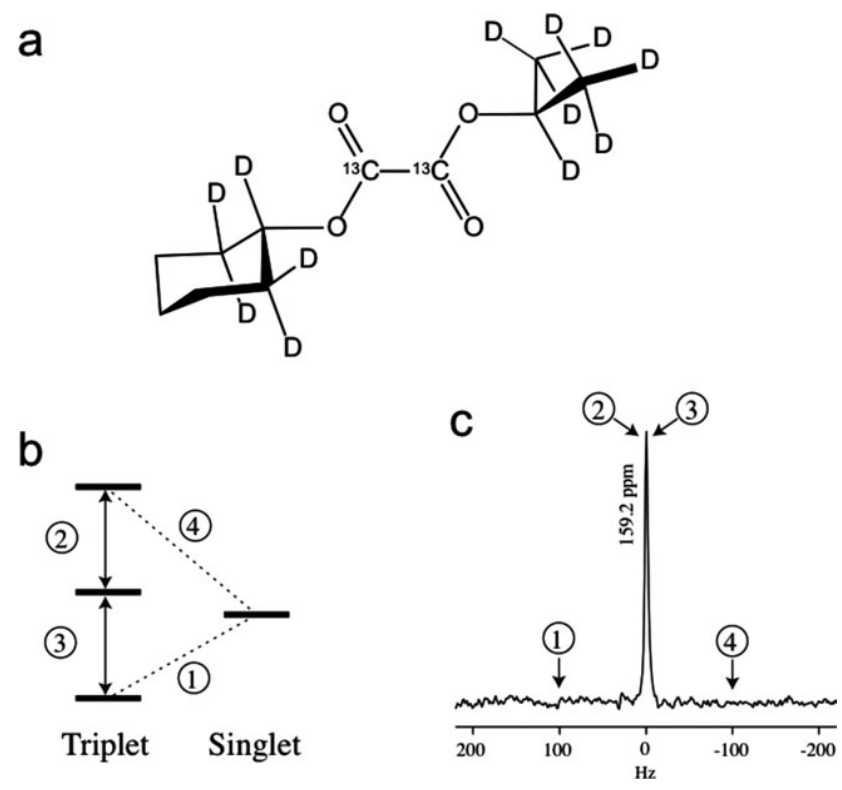

FIG. 1. a: Molecular structure of cyclohexyl-isopropyl-1,2- ${ }^{13} \mathrm{C}_{2}-$ oxalate- $\mathrm{d}_{18}$ diester; (b) energy level diagram showing singlet and triplet states and the assignments of the peaks to the spectral transitions. c: $300-\mathrm{MHz}{ }^{13} \mathrm{C}$ NMR spectrum of cyclohexyl-isopropyl-1,2- ${ }^{13} \mathrm{C}_{2}$-oxalate- $\mathrm{d}_{18}$ in methanol- $\mathrm{d}_{4}$.

energy at $94.145 \mathrm{GHz}$ for about $45 \mathrm{~min}$. The ${ }^{13} \mathrm{C}$ polarization build up was monitored via the ${ }^{13} \mathrm{C}$ solid state NMR signal (build up time constant $2500 \mathrm{~s}$ ). The sample was dissolved in $4 \mathrm{~mL}$ of methanol- $\mathrm{d}_{4}$ at 10 bar and $170^{\circ} \mathrm{C}$ in the Hypersense. The sample was collected in a syringe and manually transferred to the MR scanner in about $9 \mathrm{~s}$ for the second part of the experiment. The sample temperature after dissolution was $\sim 37^{\circ} \mathrm{C}$.

\section{Hyperpolarized NMR in the Imaging Scanner}

After transfer to the scanner, two signals were recorded (Fig. 2). The first signal $\left(S_{\mathrm{A}}\right)$ was acquired after a $10^{\circ}$ flip angle pulse to be used as a reference of the sample hyperpolarization. After this, the remaining longitudinal magnetization was converted optimally into singlet order by a $90^{\circ}$ pulse followed by a magnetization-to-singletorder pulse sequence (M2S) $(11,12)$. The singlet order was stored for some time, $\tau_{R}$, before optimal conversion back into observable transverse magnetization by applying the M2S sequence in reverse chronological order (S2M) (11,12). The resulting NMR signal is detected in the second acquisition $\left(S_{\mathrm{B}}\right)$. Note that the $\mathrm{S} 2 \mathrm{M}$ and M2S sequences are designed for the conversion of transverse magnetization into singlet order, and vice versa, as described in Ref. 11,12. The amplitude of hyperpolarized singlet order, relative to hyperpolarized magnetization, may be estimated from the amplitude ratio $\left(S_{\mathrm{B}} / \cos \left(10^{\circ}\right)\right) /$ $\left(S_{\mathrm{A}} / \sin \left(10^{\circ}\right)\right)=\left(S_{\mathrm{A}} / S_{\mathrm{B}}\right) \tan \left(10^{\circ}\right)$. This ratio corrects for the variability of the hyperpolarized longitudinal magnetization generated by the dissolution-DNP procedure. The trigonometric factors account for the conversion efficiency from longitudinal magnetization into observable coherences for the small flip angle pulse, and also the destruction of longitudinal magnetization caused by the 


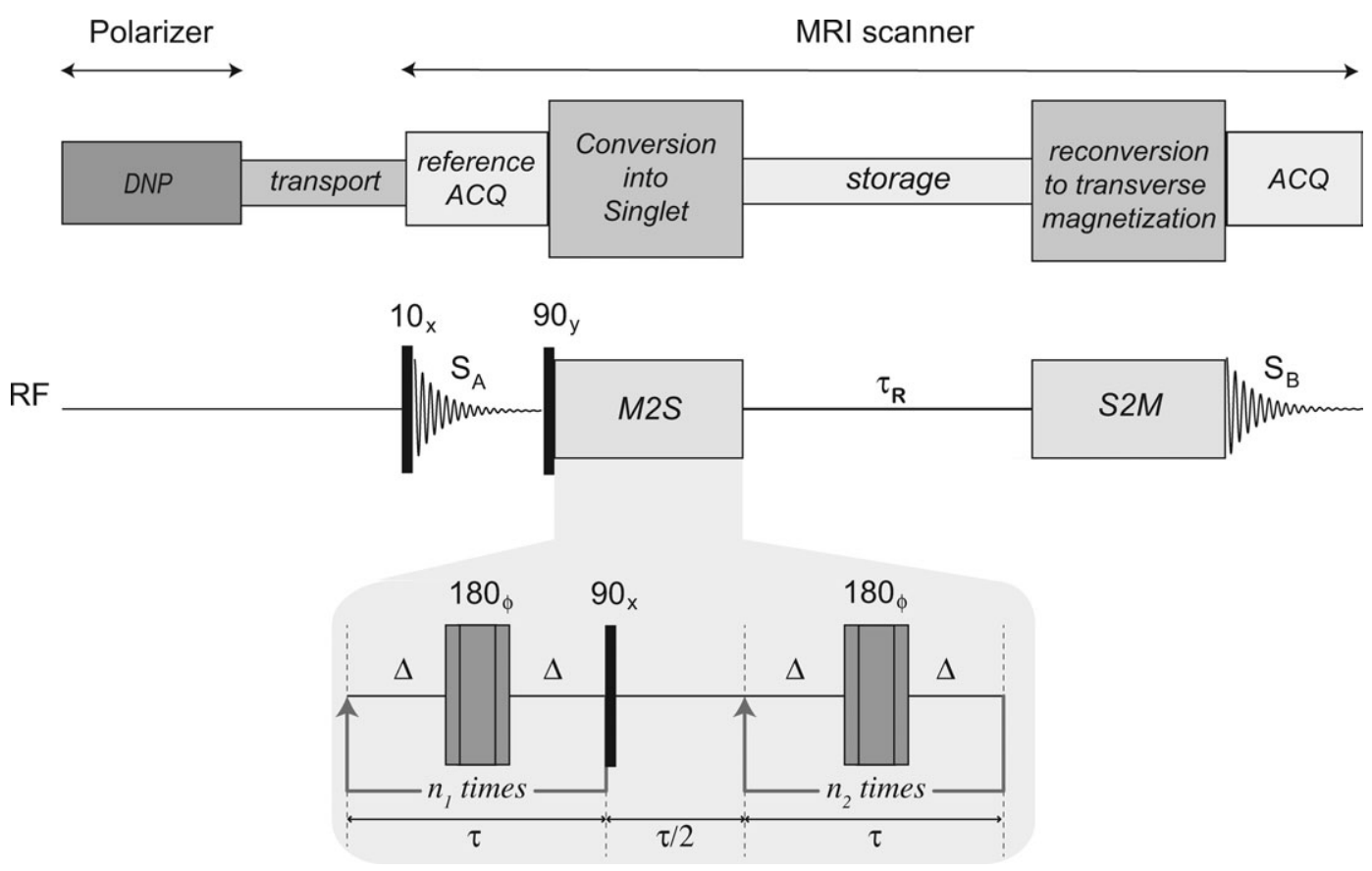

FIG. 2. Pulse sequence for measuring the lifetime of the singlet order in nearly equivalent spin pair. After dissolution-DNP, the sample is manually transferred into a preclinical 4.7 T Agilent MR scanner. A low flip angle pulse is used to measure the initial magnetization $\left(S_{A}\right)$. Immediately after the first acquisition, a 90 degree pulse is applied to produce transverse magnetization. Successively, a train of radiofrequency pulses is applied to convert transverse magnetization into singlet order (M2S). The hyperpolarized singlet order is stored in the scanner magnetic field for a variable time $\tau_{R}$ and retrieved $\left(S_{B}\right)$ by applying the mirror image of the M2S sequence (S2M). The M2S and S2M pulse sequences are as defined in Ref. 11,12 and transform transverse magnetization into singlet order, and vice versa.

same pulse. The experiment was repeated a number of times, each time extending the length of the singlet storage period, $\tau_{\mathrm{R}}$, in the $4.7-\mathrm{T}$ MR scanner magnetic field. In Figure 3, we have plotted this ratio as a function of time, $\tau_{\mathrm{R}}$, between the M2S and S2M blocks.

Figure 2 shows the pulse sequence used to demonstrate the hyperpolarization of "self-locked" singlet order and measure its long lifetime. The detailed views of M2S and S2M elements are reported in Figure 2. The M2S pulse sequence involves a set of two spin-echo trains (containing $n_{1}$ and $n_{2}$ echoes, respectively) bisected by a $90^{\circ}$ pulse. The echoes use compensated $180^{\circ}$ rotations, implemented by the composite pulse $\left[90_{90} 180_{0} 90_{90}\right]$. The total echo duration, including the finite length of the composite pulses, is given by:

$$
\tau=\tau_{\mathrm{p}}+2 \Delta=\frac{1}{2 \sqrt{J^{2}+\Delta v^{2}}}
$$

where $\Delta v$ is the chemical shift frequency difference between the two nuclear sites, $J$ is the carbon-carbon scalar coupling, $\tau_{p}$ is the composite pulse duration, and $\Delta$ is the echo interval before and after the composite pulse. Both radiofrequency amplitude and offset, errors are compensated by cycling the overall phase of the pulses through a four-step angle $\left\{0,0,180^{\circ}, 180^{\circ}, \ldots\right\}$. The pulse sequence S2M transforms singlet order back to transverse magnetization which induces an NMR signal. Further details on this sequence are reported in Ref. 12.

Values of $J$ and $\Delta v$ were determined through initial experiments on a thermally polarized sample of the oxalate. The chemical shift difference between the two carbons sites
$\Delta v=6.6 \pm 1.0 \mathrm{~Hz}$ was obtained from the spectrum of natural abundance cyclohexyl-isopropyl oxalate. An estimate of the carbon-carbon J-coupling, $J=100.3 \pm 0.5 \mathrm{~Hz}$, was obtained from the $\mathrm{AB}$ spectrum of 1-methyl-2-ethyl- $\left[{ }^{13} \mathrm{C}_{2}\right]$ oxalate ester. These measurements provide starting estimates for $n_{1}, n_{2}$, and $\Delta$, which were then empirically optimized for maximum magnetization-to-singlet conversion. The optimal parameters were found to be $\Delta=1.89 \mathrm{~ms}, n_{1}=$ 24 , and $n_{2}=12$, using nominal $90^{\circ}$ pulses of duration 300 $\mu \mathrm{s}$, providing composite pulse durations of $\tau p=1.2^{\prime \prime} \mathrm{ms}$ ".

For $T_{1}$ measurement on the hyperpolarized sample, spectra were recorded at intervals of $\tau_{\mathrm{R}}=10 \mathrm{~s}$ using $10^{\circ}$ flip angle pulses. The signal decay was fitted with a single exponential function with the effective time constant $T_{1}^{\text {eff }}$. To obtain the value of $T_{1}$, the signal depletion due to successive small flip angle pulses was corrected using:

$$
\frac{1}{T_{1}}=\frac{1}{T_{1}^{\mathrm{eff}}} \frac{\ln \left(\cos \left(10^{\circ}\right)\right)}{\tau_{\mathrm{R}}}
$$

Data processing was performed using the SpinDynamica software package (http://www.spindynamica.soton.ac.uk) for Mathematica 8 (Wolfram Research, Inc., Mathematica, Version 8.0, Champaign, IL), iNMR 4.2.3 (Nucleomatica, Molfetta, Italy), and Matlab (MathWorks, Inc., Natick, MA), with 16k point zero filling and $10 \mathrm{~Hz}$ exponential line broadening.

\section{RESULTS}

The hyperpolarized sample gave an effective longitudinal relaxation time constant $T_{1}^{\text {eff }}$ of $49 \pm 2 \mathrm{~s}$, and when 


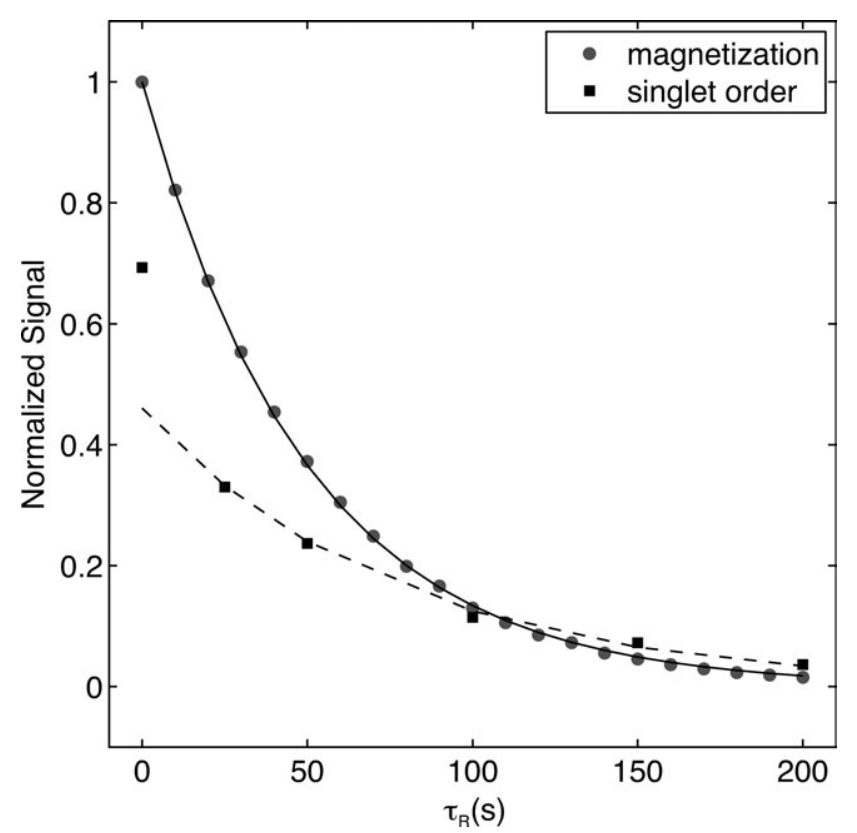

FIG. 3. Circles: cyclohexyl-isopropyl-1,2- ${ }^{13} \mathrm{C}_{2}$-oxalate- $d_{18}$ was hyperpolarized and dissolved in methanol- $\mathrm{d}_{4}$ and the NMR signal measured every $10 \mathrm{~s}$ with a $10^{\circ}$ flip angle pulse. The circles indicate the signal area as a function of time, divided by the signal area for the first time point. The solid line through the data points is a single exponential fit with time constant $\left(T_{1}{ }^{\text {eff }}\right)$ of $49 \mathrm{~s}$. Squares: six individual hyperpolarization experiments were performed on cyclohexyl-isopropyl$1,2-{ }^{13} \mathrm{C}_{2}$-oxalate- $\mathrm{d}_{18}$ in methanol- $\mathrm{d}_{4}$. In each case, a reference NMR signal was induced by a 10 degree pulse, and the remaining magnetization converted into singlet order by a 90 degree pulse followed by a M2S sequence, stored for a variable time interval, reconverted into observable magnetization by a S2M sequence, and the signal detected. The squares represent the signal intensity after the M2SS2M sequence divided by the reference signal intensity, and multiplied by the factor $\tan \left(10^{\circ}\right)$. These points represent the fraction of total hyperpolarized transverse magnetization that remains after the M2S-S2M pulse sequence using the specified storage interval. The dashed line through the data points (excluding the first point) is a single exponential fit with singlet decay time constant $\left(T_{\mathrm{S}}\right)$ of $77 \mathrm{~s}$.

corrected according to (Eq. 2), a time constant $T_{1}$ of $54 \pm$ 2 s. The experiment involving conversion between hyperpolarized magnetization and singlet order (Fig. 2) produced an intensity decay whose values at later times were fit to an exponential time constant $T_{\mathrm{S}}$ of $77 \pm 12 \mathrm{~s}$ (Fig. 3). The first point in the decay was excluded from the exponential fitting due to a strong contamination from triplet magnetization, which decays more rapidly. Such nonexponential behavior is frequently seen in this type of experiment, because the conversion of magnetization into singlet order is not $100 \%$ efficient, and generates by-product forms of spin order $(4,13)$. The theoretical maximum amplitude of singlet order generated by M2S is approximately $2 \mathrm{p} / 3$, where $p$ is the starting longitudinal polarization. The $y$-intercept of the fitted data in Figure 3 indicates an experimental efficiency of $\sim 50 \%$, which is not far from the $67 \%$ predicted by theory.

\section{Perspectives}

We have shown that it is possible to use the long lifetime of nuclear singlet states to maintain hyperpolarized order for a time greater than $T_{1}$ in a small animal imaging system. The method is simple and robust and can easily be incorporated into MRI pulse sequences as preparation pulses.

This particular molecule displays a relatively short value of $T_{S}$, despite its deliberate design. The origin of this relatively rapid singlet relaxation is currently under investigation. We believe it to be dominated by the coupling between the nuclear spins and the fluctuating molecular angular momentum (the spin-rotation mechanism). There may also be a small contribution from the electron spins of the unpaired radicals. Work is in progress for suppressing both of these relaxation contributions.

By using nearly equivalent spin pairs, we avoid the need to apply strong radiofrequency fields to inhibit singlet-triplet transitions, or to induce chemical reactions to modify the molecular symmetry. In the current form, this method is only applicable to a restricted class of molecular systems, and the demonstration system we have chosen for this preliminary study is not biocompatible. Experiments are in progress for extending these experiments to molecular systems that are of biological or metabolic importance so that they may be applied to enhance the lifetime of hyperpolarization in vivo NMR and MRI.

\section{REFERENCES}

1. Ardenkjaer-Larsen JH, Fridlund B, Gram A, Hansson G, Hansson L, Lerche MH, Servin R, Thaning M, Golman K. Increase in signal-tonoise ratio of $>10,000$ times in liquid-state NMR. Proc Natl Acad Sci USA 2003;100:10158-10163.

2. Kurhanewicz J, Vigneron DB, Brindle K, et al. Analysis of cancer metabolism by imaging hyperpolarized nuclei: prospects for translation to clinical research. Neoplasia 2011;13:81-97.

3. Carravetta M, Johannessen OG, Levitt MH. Beyond the T1 limit: singlet nuclear spin states in low magnetic fields. Phys Rev Lett 2004; 92:153003-1530034.

4. Carravetta M, Levitt MH. Theory of long-lived nuclear spin states in solution nuclear magnetic resonance. I. Singlet states in low magnetic field. J Chem Phys 2005;122:214505-214514.

5. Pileio G, Levitt MH. Theory of long-lived nuclear spin states in solution nuclear magnetic resonance. II. Singlet spin locking. J Chem Phys 2009;130:214501-214514.

6. Levitt MH. Singlet and other states with extended lifetimes. Encyclopedia of Magnetic Resonance, Vol.9. Chichester, UK: Wiley; 2010.

7. Levitt MH. Singlet nuclear magnetic resonance. Annu Rev Phys Chem 2012;63:5.1-5.17.

8. Carravetta M, Levitt MH. Long-lived nuclear spin states in high-field solution NMR. J Am Chem Soc 2004;126:6228-6229.

9. Vasos PR, Comment A, Sarkar R, Ahuja P, Jannin S, Ansermet J-P, Konter JA, Hautle P, van den Brandt B, Bodenhausen G. Long-lived states to sustain hyperpolarized magnetization. Proc Natl Acad Sci 2009;106:18469-18473.

10. Warren WS, Jenista E, Branca RT, Chen X. Increasing hyperpolarized spin lifetimes through true singlet eigenstates. Science 2009;323: 1711-1714.

11. Tayler MCD, Levitt MH. Singlet nuclear magnetic resonance of nearly-equivalent spins. Phys Chem Chem Phys 2011;13: 5556-5560.

12. Pileio G, Carravetta M, Levitt MH. Storage of nuclear magnetization as long-lived singlet order in low magnetic field. Proc Natl Acad Sci 2010;107:17135-17139.

13. Pileio G, Carravetta M, Hughes E, Levitt MH. The long-lived nuclear singlet state of $15 \mathrm{~N}$-nitrous oxide in solution. J Am Chem Soc 2008; 130:12582-12583. 